\title{
Spectrum of magnetic resonance imaging findings in a family with giant axonal neuropathy confirmed by genetic studies
}

\author{
S. Ravishankar, Gaurav Goel, Chandrajit Prasad Bernd Rautenstrauss², Atchayaram N alini ${ }^{1}$ \\ Departments of Neuroimaging and Interventional Radiology and ${ }^{1}$ Neurology, National Institute of Mental Health and Neurosciences, \\ Bangalore, India, ${ }^{2}$ University Hospital Erlangen, Institute of Human Genetics, Erlangen, Institute of Human Genetics, Medical \\ Genetics Center Munich, Germany
}

\begin{abstract}
Abstrad
Giant axonal neuropathy (GAN) is a disorder that affects both the peripheral and central nervous system with clinical and genetic heterogeneity. We describe the spectrum of magnetic resonance imaging (MRI), MR-spectroscopy (MRS), and diffusion changess in two siblings from India. In addition to the described MRI findings two unreported findings, hyperintensity signals of both the globus pallidus on $\mathrm{TI}$-weighted images and involvement of bilateral thalamus, were observed. MRS showed elevated choline peaks and reduced $\mathrm{N}$-acetylaspartate peaks as well as presence of lactate peaks. Diffusion weighted images showed restricted diffusion.
\end{abstract}

Address for correspondence:

Dr. Atchayaran Nalini,

Department of Neurology, National

Institute of Mental Health and

Neurosciences, Hosur Road,

Bangalore - 560 029, India.

E-mail: atchayaramnalini@ yahoo.

co.in

DOI: $10.4103 / 0028-3886.51290$
Key words: AluYa5, giant axonal neuropathy, magnetic resonance imaging, M R-spectroscopy

\section{Introduction}

Giant axonal neuropathy (GAN) is a rare autosomal recessive disorder that affects peripheral and central nervous systems $(\mathrm{CNS})^{[1-8]}$ with both clinical and genetic heterogeneity ${ }^{[9,10]}$ The associated clinical features include sensorimotor neuropathy and varying CNS involvement including a motor neuron syndrome. ${ }^{[1-8]} \mathrm{A}$ variety of magnetic resonance imaging (MRI) abnormalities ${ }^{[6,8,11]}$ and MR-spectroscopy (MRS) and diffusion changes ${ }^{[12,13]}$ have been reported. We studied these changes in two siblings with typical clinical manifestations suggestive of GAN and confirmed by histopathological examination and by genetic analysis.

\section{$M$ aterials and M ethods}

\section{Clinical report}

A 10-year-old boy (Proband) had delayed motor and mental milestones, progressive clumsiness of gait, weakness and wasting of distal muscles of upper and lower limbs, and progressive decline in cognitive functions with dullness, apathy, and urinary incontinence. The younger brother was also evaluated at an age of six years. He had symptoms of gait ataxia, bilateral nystagmus, and mild cerebellar ataxia with absent deep tendon reflexes.

\section{Pathological studies}

The sural nerve biopsies of both siblings revealed several giant axons in all the funicles, enclosed by thinned-out myelin sheaths. Some of the bloated axons were totally denuded of myelin, and an occasional large, loose 'onion bulb' schwann cell hyperplasia was seen. Axillary skin biopsy was performed in both the brothers and the elder sibling showed enlarged axons in the dermal nerve twigs. Electron microscopy showed enlarged axons in the sural nerve biopsies, which were tightly packed with intermediate filaments displacing the other organelles to the periphery. Sural nerve biopsies showed the characteristic presence of giant axonal swelling filled with neurofilaments in both patients.

\section{Electrophysiological studies}

Visual evoked potential (VEP) showed prolonged P100 latencies. Brainstem auditory evoked response (BAER) showed increased interpeak latencies. EEG showed diffuse slowing. Nerve conduction studies revealed markedly 
reduced motor and sensory conduction velocities. EMG revealed changes suggestive of chronic reinnervation.

\section{Genetic studies}

For the proband in the family, sequence analysis revealed a homozygous insertion (c.1657ALUYa5ins, p. Thr553_Pro597del) in exon 11 of the GAN gene. The AluYa5 insertion was identified homozygous in both siblings, whereas the parents were heterozygous carriers of this mutation. This insertion starts at nucleotide position c. 1657 and includes $281 \mathrm{bp}$ of Alu Ya5 as well as an adenosine tail of at least $40 \mathrm{bp}$.

\section{Magnetic resonance imaging findings}

Case 1 (Proband): MRI revealed diffuse confluent symmetrical white matter hyperintensities on T2-weighted (T2W) and fast-fluid attenuated inversion recovery (FLAIR) sequences, which are isointense on T1W sequences involving bilateral deep periventricular white matter in frontal and parietoccipital lobes [Figure 1a-d]. It extended to involve bilateral external capsule, midbrain, pons, middle cerebellar peduncles, and bilateral cerebellar white matter [Figure 1c]. Corpus callosum was spared [Figure 1f]. Multiple focal discrete lesions hyperintense on T2W and FLAIR sequences, and isointense on T1W sequences were noted in bilateral frontal and parietal subcortical white matter [Figure 1b]. Bilateral thalamus in posteromedial region showed hyperintensity on T2W and FLAIR sequences, and isointensity on T1W sequences [Figure 1d-e]. Globus pallidus showed peripheral hyperintensity on $\mathrm{T} 1 \mathrm{~W}$, T2W, and FLAIR sequences with sparing of central
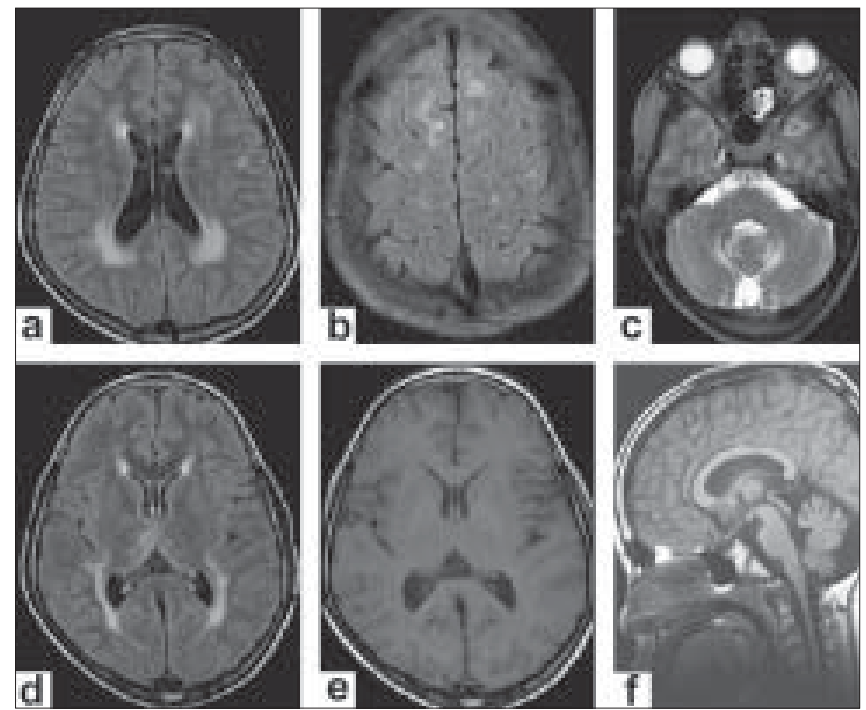

Figure 1: (a,d) White matter hyperintensities on FLAIR involving bilateral deep periventricular white matter in frontal and parietoccipital lobes (a,d); (b) Multiple focal lesions hyperintense on FLAIR noted in bilateral frontal, parietal subcortical white matter; (c) Pons, middle cerebellar peduncles, bilateral cerebellar white matter in T2W sequence;

(d) Bilateral thalamus showing hyperintensity on FLAIR; (e) Isointensity on T1W sequences; Globus pallidus shows peripheral hyperintensity on T1W (e) and FLAIR (d) with sparing of central region; (f) Cervical cord atrophy seen in $\mathrm{T} 1 \mathrm{~W}$ sagittal sequence region [Figure 1d]. Cerebral and cerebellar atrophy was present. Cervical cord atrophy was also noted [Figure 1f].

Case 2 (Younger sibling): MRI revealed diffuse confluent symmetrical white matter hyperintensities on $\mathrm{T} 2 \mathrm{~W}$ and FLAIR sequences, isointense on T1W sequences involving white matter in bilateral frontal lobes [Figure 2a-b], pons, middle cerebellar peduncles, and bilateral cerebellar white matter [Figure 2c]. Corpus callosum and subcortical fibres werespared [Figure 2f]. Bilateral globus pallidus were diffusely hyperintense on T1W, T2W, and FLAIR sequences with no loss of volume [Figure $2 \mathrm{~d}-\mathrm{e}$ ].

\section{Magnetic resonance spectroscopy}

On visual inspection, elevated choline peaks and reduced NAA peaks as well as presence of lactate peaks in supratentorial and cerebellar white matter [Figure 3b-d] were observed. Quantitative analysis showed raised Cho/NAA ratio, reduced NAA/Cr ratio, and raised Cho/ $\mathrm{Cr}$ as seen in Table 1 .

\section{Diffusion weighted imaging}

DWI revealed hyperintensity in cerebral and cerebellar white matter on b500 and hypointensity on b1000 [Figure $4 \mathrm{a}$ and $4 \mathrm{~b}$ ] with the resultant hyperintensity to white matter on ADC maps [Figure 4c and 4d]. Table 2 shows ADC values between abnormal and normal white matter with increased ADC within in the lesions (>1).

Table 1: Cerebral proton metabolite ratios of the patient diagnosed with giant axonal neuropathy

\begin{tabular}{lllll}
\hline & Cho/NAA & Cho/Cr & NAA/Cr & Lactate \\
\hline Supratentorial & 1.45 & 1.17 & 0.80 & Present \\
Cerebellar & 2.34 & 1.84 & 0.78 & Present \\
\hline
\end{tabular}
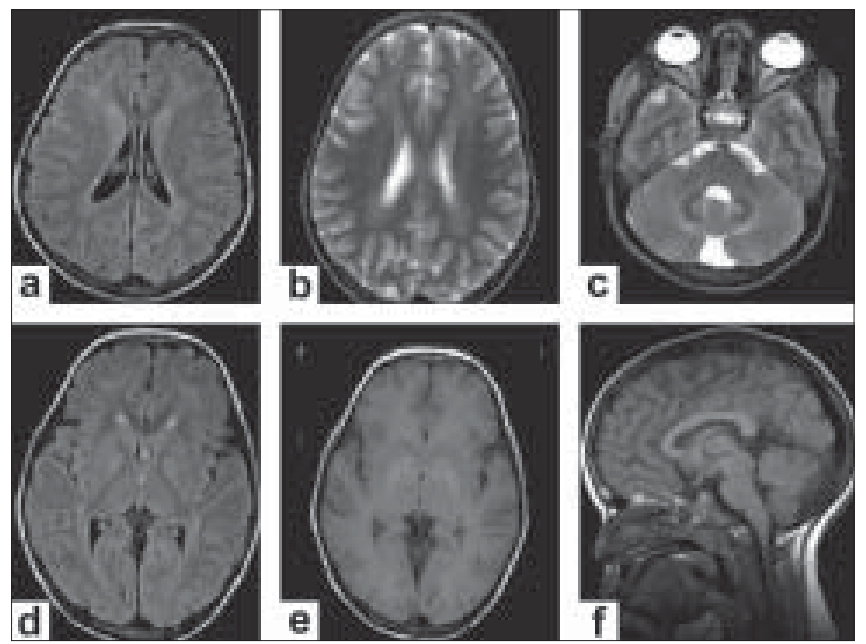

Figure 2: Bilateral frontal white matter hyper intensities on FLAIR (a) and T2W (b) Sequences; (c) White matter hyperintensities on T2W sequence in pons, middle cerebellar peduncles, and bilateral cerebellar white matter. Bilateral globus pallidus are diffusely hyperintense on FLAIR (d) and T1W (e) sequences with no loss of volume. (f) Corpus callosum is spared 

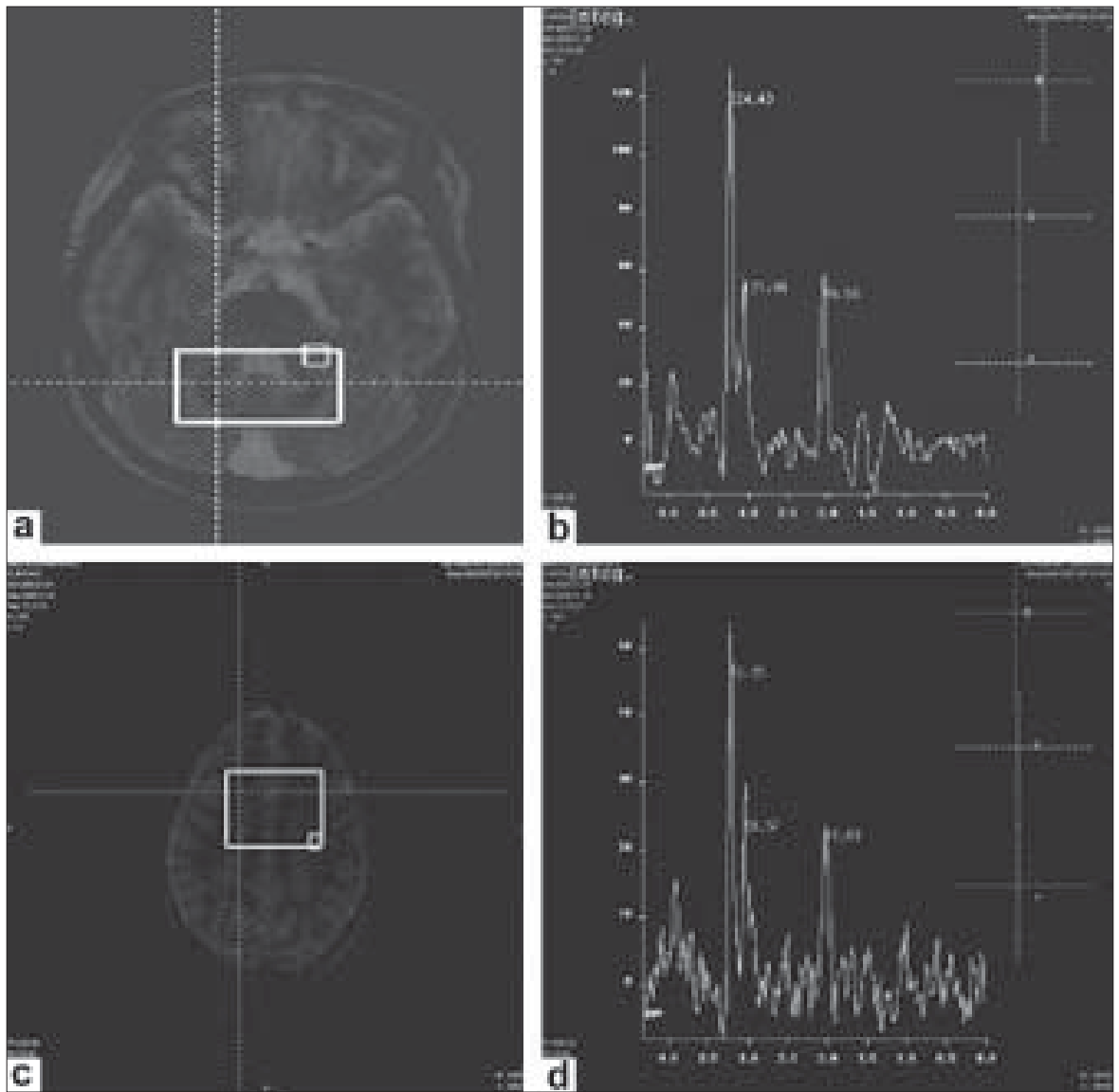

Figure 3: Magnetic resonance spectroscopy showing elevated choline peaks and reduced NAA peaks in supratentorial (d) and cerebellar white matter (b). Lactate peaks are seen in cerebellar white matter (b)

\section{Discussion}

Both the siblings in this study had classical clinical features of GAN and the diagnosis was confirmed by histopathology and genetic analysis. MRI findings showed widespread spectrum of changes, which are reported to be present in this rare autosomal recessive disease. In the elder sibling diffuse changes in frontal and parietoccipital white matter along with focal subcortical white matter changes were observed, while in younger sibling there was a predominant frontal gradient with sparing of subcortical U fibres. Corpus callosum was spared in both cases. Demir et al., have reported diffuse white matter lesions in anterior and posterior periventricular white matter, ${ }^{[8]}$ Gordon et al., reported in parietoccipital white matter, ${ }^{[14]}$ while Thomas et al., and Richen et al., described preferential involvement of frontal and parietal white matter. ${ }^{[1,15]}$ In nearly all reported cases sparing of subcortical fibres were seen, except in one case reported by Malandrini et al., where subcortical involvement was present in a 17-year-old girl ${ }^{[16]}$ with GAN. Corpus callosum thinning is reported in histopathological and imaging data ${ }^{[6,11,15,17]}$ but these were not noted in our patients.

\begin{tabular}{|c|c|c|c|}
\hline & \multicolumn{2}{|c|}{ ADC abnormal } & \multirow{2}{*}{$\begin{array}{l}\text { ADC normal } \\
\text { white matter }\end{array}$} \\
\hline & Right & Left & \\
\hline Supratentorial & 117 & 119 & 67 \\
\hline Cerebellar & 123 & 120 & 73 \\
\hline
\end{tabular}

Signal intensity changes were found in bilateral posteromedial thalamus and peripheral region of globus pallidus in the elder child. Diffuse involvement of globus pallidus without involvement of thalamus was seen in younger sibling. Demir et al., have reported a single patient with posteromedial thalamus involvement. ${ }^{[8]}$ There are no reports in the literature on involvement of globus pallidus in GAN. In our patient globus pallidus distinctly showed hyperintensity on T1W sequence while remaining white matter and thalamus showed isointensity on T1W images. Among the reports on MRI findings presence of T1 hyperintense signal abnormalities have not been described. This hyperintense signal abnormality on T1W images could possibly indicate accumulation of neurofilaments in cases of GAN, leading to T1 shortening and thus appearing hyperintense on short TR/TE sequences or it could be due to active demyelination process with breakdown of 

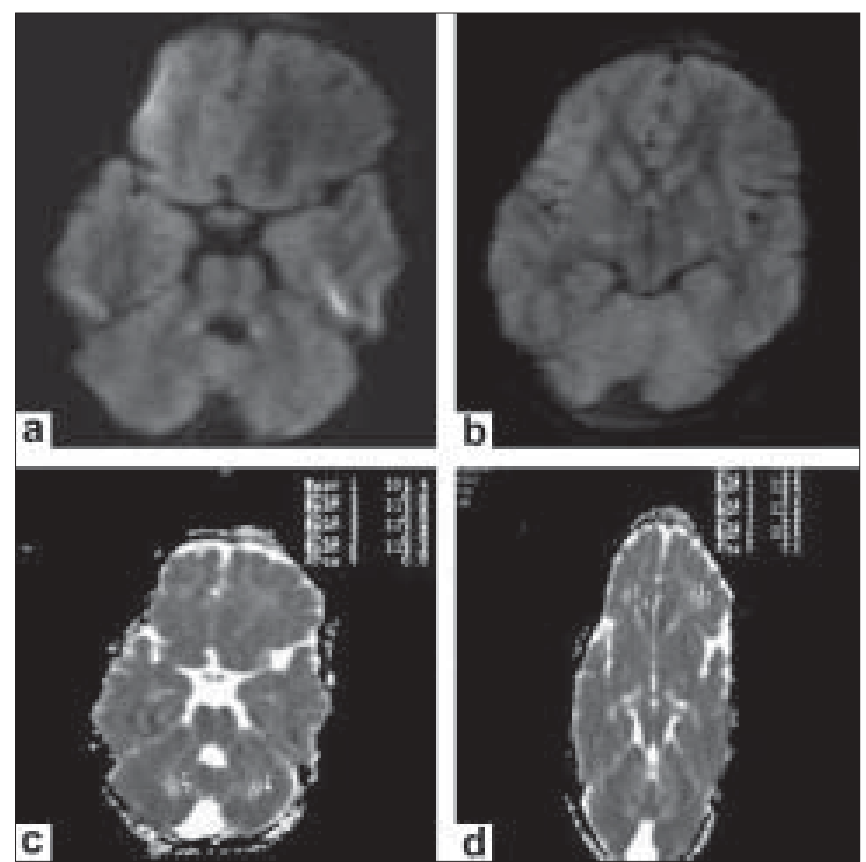

Figure 4: Diffusion weighted images showing hypointensity on b1000 in cerebellar and supratentorial white matter $(a, b)$ and resultant hyperintensity to white matter on ADC maps (c, d)

myelin, leading to $\mathrm{T} 1$ shortening or possibly combination of both. Earlier reports have shown hypointensity on T1W images, which could signify disease progression leading to gliosis and cavitation formation. . $^{[6,8,11,18]}$ Cerebellar and middle cerebellar peduncle involvement was seen in both the cases. Cerebellar and brainstem involvement was present in nearly all reported cases except for one case in a series reported by Demir et al. ${ }^{[8]}$ Marked cerebellar and cervical cord atrophy was seen in elder child but not noted in younger sibling. Richen et al., described cervical cord atrophy in one of their cases. ${ }^{[8]}$

MRS shows marked reduction of NAA and elevation of choline and presence of lactate peaks. Elevation of choline peaks and lactate suggests active demyelination ${ }^{[18]}$ and particularly presence of lactate peaks is suggestive of anaerobic metabolism by macrophages during acute phase. Reduction in NAA suggests neuronal loss. Alkan et al., ${ }^{[12]}$ reported no decrease in NAA/Cr ratios but only increase in Cho/ $\mathrm{Cr}$ ratios. MRS ratios showed that there is more severe involvement in cerebellum as compared with supratentorial white matter, which is in accordance with pathological data, which shows predominant involvement of cerebellar white matter. ${ }^{[15,17]}$

Increased apparent diffusion coefficient values in white matter suggests increased mobility of water molecules in the brain of patients with GAN ${ }^{[8]}$ This finding indicates a myelin disorder such as demyelination and when viewed in combination with MRS data reflects neuronal damage, active demyelination, and glial proliferation in accordance with postmortem data. ${ }^{[3]}$ Thus, the spectrum of findings in MRI signifies wide variation in disease affection and subclinical MRI changes and along with MRS and DWI provides an in vivo assessment of pathological alterations in patients with GAN. In conclusion, genetic heterogeneity and involvement of various structures in the central nervous system possibly leads to varied MRI features in GAN. Hyperintensity of the lesions on T1W MR images, which may probably be due to accumulation of neurofilaments is the most characteristic and consistent feature which can lead to the diagnosis of this entity.

\section{References}

1. Asbury AK, Gale MK, Cox SC, Baringer JR, Berg BO. Giant axonal neuropathy: A unique case with segmental neurofilamentous masses. Acta Neuropathol (Berl) 1972;20:237-40.

2. Berg BO, Rosenberg SH, Asbury AK. Giant axonal neuropathy. Pediatrics 1972;49:894-9.

3. Carpenter S, Karpati G, Andermann F, Gold R. Giant axonal neuropathy a clinically and morphologically distinct neurological disease. Arch Neurol 1974;31:312-6.

4. Igisu H, Ohta M, Tabira T, Hosokawa S, Goto I, Kuroiwa Y. Giant axonal neuropathy. A clinical entity affecting the central as well as the peripheral nervous system. Neurology 1975;25:717-21.

5. Ouvrier RA. Giant axonal neuropathy: A review. Brain Dev 1989;11 207-14.

6. Lampl Y, Eshel Y, Ben-David E, Gilad R, Sarova-Pinhas I, Sandbank U. Giant axonal neuropathy with predominant central nervous system manifestations. Dev Med Child Neurol 1992;34:164-9.

7. Bruno C, Bertini E, Federico A, Tonoli E, Lispi ML, Cassandrini D, et al. Clinical and molecular findings in patients with giant axonal neuropathy (GAN). Neurology 2004;62:13-6.

8. Demir E, Bomont P, Erdem S, Cavalier L, Demirci M, Kose G et al. Giant axonal neuropathy: Clinical and genetic study in six cases. J Neurol Neurosurg Psychiatry 2005;76:825-32.

9. Boltshauser E, Bischoff A, Isler W. Giant axonal neuropathy, report of a case with normal hair. J Neurol Sci 1977;31:269-78

10. Peiffer J, Schlote W, Bischoff A, Boltshauser E, Muller G. Generalized giant axonal neuropathy: A filament-forming disease of neuronal, endothelial, glial and Schwann cells in a patient without kinky hair. Acta Neuropathol (Berl) 1977;40:213-8.

11. Richen P, Tandan R. Giant axonal neuropathy: Progressive clinical and radiologic CNS involvement. Neurology 1992;42:2220-2.

12. Alkan A, Kutlu R, Sigirci A, Baysal T, Altinok T, Yakinci C. Giant axonal neuropathy: MRS findings. J Neuroimaging 2003;13:371-5.

13. Alkan A, Sigirci A, Kutlu R, Doganay S, Erdem G, Yakinci C. Giant axonal neuropathy: Diffusion weighted imaging features of the brain. J Child Neurol 2006;21:912-5.

14. Neil G. Giant axonal neuropathy: A review. Dev Med Child Neurol 2004;46:717-9.

15. Bomont P, Cavalier L, Blondeau F, Ben Hamida C, Belal S, Tazir M, et al. The gene encoding gigaxonin, a new member of the cytoskeletal $\mathrm{BTB} /$ kelch repeat family, is mutated in giant axonal neuropathy. Nat Genet 2000;26:370-4.

16. Malandrini A, Dotti MT, Battisti C, Villanova M, Capocehi G, Federer A. Giant axonal neuropathy with subclinical involvement of the central nervous system: A case report. J Neurol Sci 1998;158:232-5.

17. Kretzschmar HA, Berg BO, Davis RL. Giant axonal neuropathy: A neuropathological study. Acta Neuropathol (Berl) 1987;73:138-44.

18. Brockman K, Pouwels PJ, Dechent P, Flanigan KM, Frahm J, Hanefeld F. Cerebral proton magnetic resonance spectroscopy of a patient with giant axonal neuropathy. Brain Dev 2003;25:45; 25:45-50.

Accepted on 31-03-2009

Source of Support: Nil, Conflict of Interest: None declared. 